\title{
Characterisation of nanomaterials in biological samples
}

\author{
Heidi Goenaga-Infante • Erik H. Larsen
}

Published online: 20 April 2014

(C) Springer-Verlag Berlin Heidelberg 2014

The novel properties of nanomaterials and their increasing use have led to enormous research and development activity and significant markets of consumer products containing nano-objects. Many of the nano-objects used in these products have been incorporated intentionally or occur accidentally. Nano-objects are defined as having at least one dimension between $1 \mathrm{~nm}$ and $100 \mathrm{~nm}$ (ISO/TS 80004-1) and this is the primary criterion that makes them "nano." Examples of nano-objects are those with metal cores (e.g., metals, metals oxides, quantum dots, alloys, and core-shell materials), and selected carbonaceous nano-objects (e.g., carbon nanotubes). Examples of biological matrices where nanomaterials are used include food, food packaging, cosmetics, pharmaceuticals, nutraceuticals, etc.

Although there is concern about the possible toxic effects of nanomaterials because of their unique properties, there is no definitive proof for toxicity. In vitro nanotoxicology studies, in the short term, and the quantification of human exposure, in the long term, will require metrologically validated methods for the detection and characterisation of

Published in the topical collection Characterisation of Nanomaterials in Biological Samples with guest editors Heidi Goenaga-Infante and Erik H. Larsen.

H. Goenaga-Infante $(\bowtie)$

LGC Ltd, Queens Road Teddington, Middlesex TW11 OLY, UK

e-mail: heidi.goenaga-infante@lgc.co.uk

E. H. Larsen

Department of Food Chemistry, Technical University of Denmark, National Food Institute, Mørkhøj Bygade 19, 2860 Søborg, Denmark e-mail: ehlar@food.dtu.dk manufactured nano-objects in complex matrices. Such methods are scarce and, therefore, urgently needed to support upcoming regulation and enable quality control of existing products.

Efforts to establish regulations and guidelines related to the use and characterisation of nanomaterials have increased over the last 5 years. The EU Cosmetic Product Regulation (1223/2009) currently defines a cosmetic nanomaterial ingredient as follows: 'nanomaterial' means an insoluble or biopersistant and intentionally manufactured material with one or more external dimensions, or an internal structure, on the scale from 1 to $100 \mathrm{~nm}$. Recommendations from the EU Commission published in October 2011 (2011/696/EU) stated that for regulatory purposes, the size distribution of a material should be presented as size distribution based on the number concentration (i.e., the number of objects within a given size range). This recommendation is in contrast to the mass fraction of nanoscale particles in the nanomaterial, as a small mass fraction may contain the largest number of particles. In 2011, FDA released guidelines for industry (2011-D-04890002) with regards to the use of nanoparticles as food additives. The British Standards Institute has recently released guidelines (PAS 139:2012) on the detection and characterisation of manufactured nano-objects in complex matrices. These definitions, recommendations, and guidelines suggest that proper characterisation of nanomaterials in food and consumer products etc. is a demanding task.

Analytical techniques that have potential for the characterisation of nanomaterials in biological samples include fractionation techniques such as field flow fractionation, size exclusion chromatography, hydrodynamic chromatography, dialysis, filtration, and ultrafiltration, and detection 
techniques such as imaging by transmission electron microscopy (TEM), elemental detection by ICP-MS, ICP$\mathrm{OES}$, and AAS, atomic force microscopy (AFM) for structure, and light scattering for sizing. For example, ICP-MS has been used to quantify total elemental and isotopic composition and ratio, along with particle number concentration, whereas electron microscopy is used to measure size distribution, aggregation, shape, crystal structure, and, with associated spectroscopy methods, elemental concentration and speciation. Amongst the range of techniques available for nanomaterial characterisation, field-flow fractionation in combination with elemental and sizing detectors has emerged over the last decade as a highly promising approach for size-based detection of nanomaterials in complex samples. Whilst there are already several examples of using FFF-ICP-MS for nanomaterials in environmental samples, the literature dealing with NPs in biological materials is relatively scarce. Given the complexity of samples containing nano-objects in most biological matrices, the use of a single measurement technique has often resulted in their ambiguous detection and characterisation. The use of multi-method approaches based on separation by FFF with appropriate off- or on-line detectors has, therefore, been proven to be essential in providing improved information on size, size distribution and sized-based elemental composition in suspensions, and reduced bias, especially for nanoobjects in complex matrices.

For most biological applications, a remaining critical challenge is the extraction of nano-objects from the sample matrix into a suspension without causing particle transformation. Another major challenge is preserving the stability of nano-objects in suspensions and during analysis. For example, losses of nano-objects due to dissolution, adsorption on containers, stationary phases, cut-off membranes, or other filtering devices are likely to occur during sample handling and analysis, so they should be minimized or controlled. Temperature changes, exposure to light and/or increase of sample turbulence may affect the property of both the nano-object and the matrix by enhancing their aggregation and photo-oxidation and by increasing the microbiological activity of the matrix. Unfortunately, only few papers reporting a systematic development considering these factors have been published so far. A contributing factor is the lack of like-for-like standards or certified reference materials, which is a remaining problem, currently affecting quality assurance of the measurements in the field of analysis of nanomaterials in biological materials.
In an effort to support the expected upcoming regulation, the development of methods that are able to determine size distribution based on the number concentration has become the focus of expert groups on the field. Methods currently available to determine particle number such as nanoparticle tracking analysis (NTA) and the microscopy techniques transmission and scanning electron microscopy (TEM and SEM) and atomic force microscopy (AFM) can characterise nanoparticles in terms of size and size distribution, but they are often compromised by the presence of a complex matrix and/or are very time consuming. Single particle analysis by ICP-MS (sp ICP-MS) is emerging as a promising tool for nanoparticle sizing and counting, thanks to recent improvements in ICP-MS instrumentation making shorter dwell times and improved detection sensitivity possible. Although the number of successful publications dedicated to sp ICP-MS has dramatically increased over the past 5 years, there are remaining challenges associated with sample matrix dilution and the lower limit of size detection, which currently is around $20 \mathrm{~nm}$ for pure metallic nanomaterials' possible aggregate formation. Therefore, alternative ways to reduce the matrix (e.g., by enzymatic or acid hydrolysis) without changing the nanomaterial are currently under investigation. Finally, applications of this approach to real complex matrices have also shown the need for production of like-for-like well-characterised nanomaterial standards for method optimisation (e.g., calibration of nebulization efficiency) in order to achieve an accurate number-based size distribution.

This topical issue comprises seven original papers. Together, these contributions highlight the complexity and role of nanomaterial characterisation in providing information relevant to consumer product safety and toxicology. The articles cover a range of nanomaterials of different types (e.g., gold, silver, silica, titania, selenium, and organic micelle-type nanoparticles as well as carbon nanotubes) and biological samples of different complexity (e.g., animal tissue, food, cells). Of particular interest is the inclusion of papers on reference material production and interlaboratory comparisons of nanomaterial characterisation measurements. They are essential for future quality assurance and method validation. We hope that this collection of excellent papers will help to broaden the horizons for future developments in this field. We would like to thank all the authors for their contributions and hope that you enjoy reading them. 


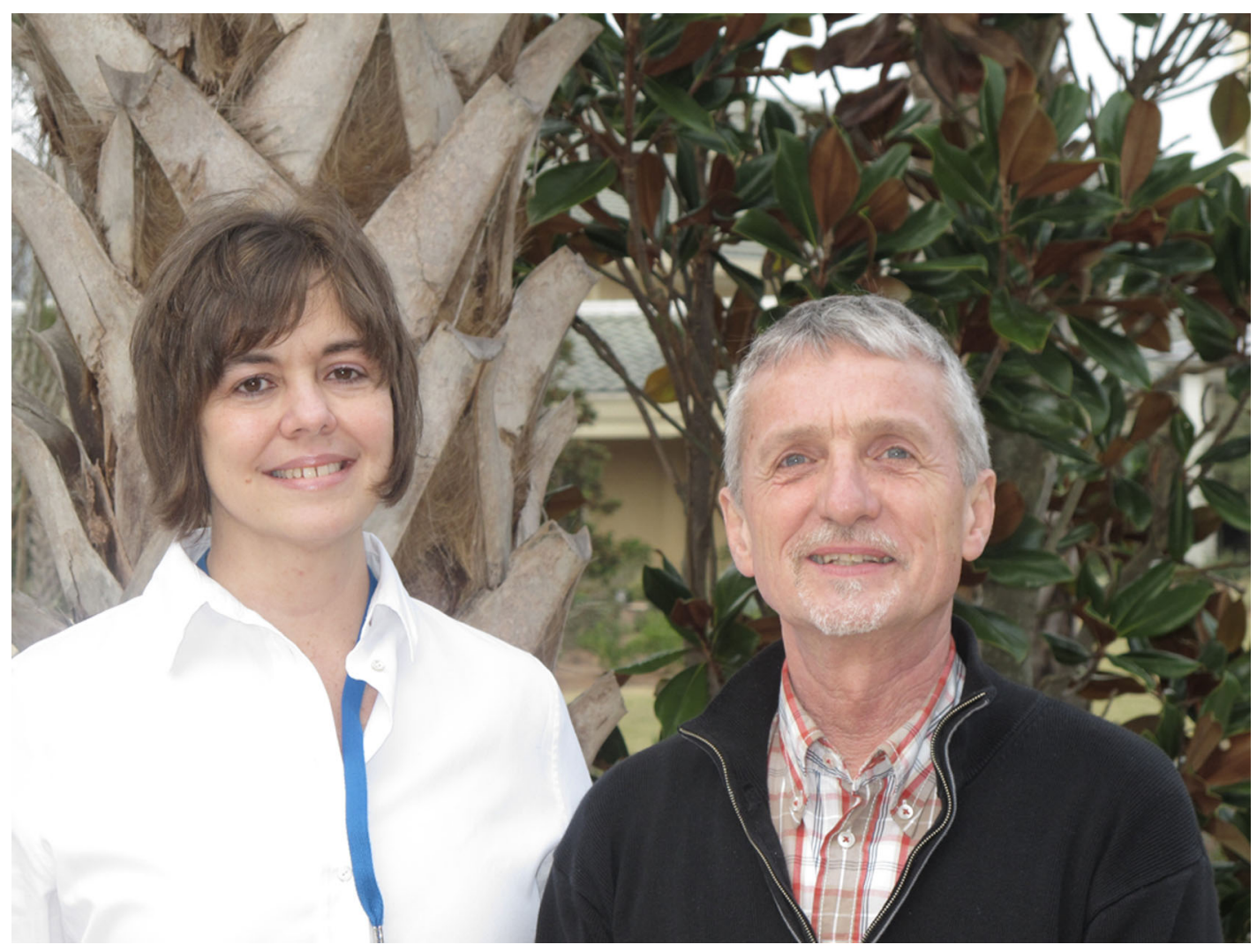

Heidi Goenaga-Infante (left) and Erik H. Larsen (right)

Heidi Goenaga Infante is currently Principal Scientist and Team Leader of the Inorganic Analysis Team within the Science and Technology division at LGC Ltd. Heidi's scientific career of over 15 years of experience on elemental and speciation analysis started with the award of a $\mathrm{PhD}$ from Oviedo University, Spain. Her current research interests and expertise lie in trace element speciation analysis, metallomics research, combined use of elemental and molecular mass spectrometry, size-based element fractionation and the characterisation of "speciated" reference materials and standards. Dr Goenaga Infante is the UK representative at the Inorganic Analysis Working Group of the CCQM, the International Consultative Committee for Metrology in Chemistry. She is also the coordinator of the European Metrology Research Project "Chemical and Optical Characterisation of Nanomaterials in Biological Systems". Heidi is a member of the international advisory boards of Analytical and Bioanalytical Chemistry, the RSC journals JAAS and Metallomics, and a member of IUPAC.
Erik H. Larsen is Senior Researcher and Group Leader of the Metals, Minerals, and Nanomaterials Team of The National Food Institute, Technical University of Denmark. Erik received his Ph.D. degree from the University of Copenhagen in 1993, and has been one of the pioneers of speciation analysis (arsenic, selenium, mercury). In 2007, Erik initiated research on nanoparticles in food and biological samples obtained from projects within nanotoxicology. He established an analytical platform based on asymmetric FFF with on-line detectors including UV, FL, MALS, DLS, and ICP-MS. Erik has received ample external funding from national and international sources, and is an active partner in nanometrology projects such as NanoLyse, NanoDefine, and NanoSolutions, which are funded by the European Union. He is a member of the international advisory board of Analytical and Bioanalytical Chemistry. 